\title{
The Effect of a Traditional Dance Program on Health-Related Quality of Life as Perceived by Primary School Students
}

\author{
Lykesas Georgios ${ }^{1}$, Giosos Ioannis ${ }^{2}$, Theocharidou Olga ${ }^{1}$, Chatzopoulos Dimitris ${ }^{1}$, Koutsouba Maria ${ }^{2}$ \\ ${ }^{1}$ School of Physical Education and Sport Science, Aristotle University of Thessaloniki, Greece \\ ${ }^{2}$ School of Physical Education and Sport Science, National and Kapodistrian University of Athens, Greece \\ Correspondence: Lykesas Georgios, School of Physical Education and Sport Science, Aristotle University of \\ Thessaloniki, Greece.
}

Received: November 26, 2017

Accepted: December 11, 2017 Online Published: December 22, 2017

doi:10.11114/jets.v6i1.2878

URL: https://doi.org/10.11114/jets.v6i1.2878

\begin{abstract}
Physical exercise is acknowledged to play a highly significant role in human health and life quality. The concept "Quality of Life" (QoL) refers to many parameters, including the individual's physical condition, psychological status, level of independence, social relationships and interaction with the environment. Participating in dance activities, and more particularly in traditional dances, can significantly increase one's physical and psychological well-being, and therefore improve their QoL.

The aim of the present study was to investigate whether there are differences in the way Health-Related Quality of Life (HRQoL) is perceived by fifth and sixth graders of two public primary schools attending two different programs: a) program focused on traditional dance and b) program implemented within the context of the Physical Education curriculum as provided by the Ministry of Education, Research and Religious Affairs.

According to the results of the research, the traditional dance intervention program presented higher perceived HRQoL values in six of the eight dimensions of the Kidscreen-52 Questionnaire, these dimensions being "Health", "Mood", "Self-perception", "Friends", "School-Learning" and "Self \& Others". The most highly statistically significant difference was found for the "Health" dimension.

In conclusion, traditional dance offers multiple benefits to participants, since it is an enjoyable physical activity contributing to the improvement of many QoL-related factors.
\end{abstract}

Keywords: Quality of Life (QoL), Health-Related Quality of Life (HRQoL), traditional dance, primary school

\section{Introduction}

Given that Quality of Life (QoL) has a different meaning for every individual, there are different approaches to its definition. The relationship between $\mathrm{QoL}$ and physical exercise is a research field with continuously increasing interest. In recent years, this relationship has been thoroughly investigated suggesting dance as a form of exercise suitable for all ages as it seems to have a particularly beneficial effect on both physical and mental health (Lykesas, \& Zachopoulou, 2006; Lykesas, et al., 2010; Bougiesi, et al., 2014; Lykesas, et al., 2017). During the last decades, there has been intense research interest in the concept of "Quality of Life" (QoL) and QoL measurement methods (Tountas et al., 2006). The conceptual definition of QoL within the context of economic, social, psychological and medical sciences (Tyrovola et al., 2011; Petraki \& Koutsouba, 2013, Venetsanou \& Koutsouba, 2015) indicates its multiple and diverse domains (Cummins, 2005) while at the same time highlights both its objective and subjective dimension (Economou et al., 2001).

According to one of the first approaches, QoL was defined as one's sense of well-being and satisfaction with life, and their feeling of happiness regarding their health condition, energy and stress levels, goal-setting, self-esteem, depression, social and family support (Dalkey \& Rourk, 1973). Even from this very first definition it became clear that QoL includes elements related to both physical and mental health (Chodzko-Zajki, 2005). As a consequence, the term "Health-Related Quality of Life" (HRQoL) was later on introduced, referring mostly to health assessment in its broader sense (Chen, $\mathrm{Li}, \&$ Kochen 2005), and was recognized as a field of QoL focusing on the assessment of physical, mental and social well-being (Mooney, 2006). Bullinger (2003) defines HRQoL as a multi-dimensional concept comprising 
domains related to physical/ mental/ emotional/ social functioning and well-being as perceived by the individual. In other words, HRQoL is a broad multifactorial concept that keeps changing depending on the individual's health condition, relationships, experiences and roles.

HRQoL measurements can help: (a) determine the stress burden resulting from poor health in a population, (b) identify subgroups where HRQoL has unmet needs, (c) inform about the development of targeted interventions and d) monitor changes in HRQoL that may potentially occur over time (Jiang \& Hessel, 2006). In particular with regard to children, HRQoL measurements can provide information for protecting children's health. Such measurements have been conducted in Greece (Tountas et al., 2006; Karasimopoulou et al., 2009) while surveys investigating children's HRQoL in relation to Greek traditional dance have been carried out only within the context of Cultural Associations (Darginidou $\&$ Goulimaris, 2016) and not within the context of School Physical Education.

\subsection{Health-Related Quality of Life (HRQoL) \& Dance}

Following a literature review, there are several studies for adults and much fewer for children that investigate the effect of dance interventions on HRQoL. A pilot study in 35 women was conducted at 2 Cancer Centers in Connecticut to determine the effect of a Dance and Movement Program on QoL and shoulder function of breast cancer survivors. The study showed that the dance and movement program significantly improved the patients' shoulder function while increasing their feeling of well-being and satisfaction for life, suggesting this form of therapy as part of continuing care for breast cancer survivors (Sandel et al, 2005). According to literature, dance helps reduce stress, enhances self-knowledge, self-esteem, well-being and positive mood while being a good type of exercise for improving and/ or maintaining physical fitness and health (Doulias et al., 2005). A similar study upon motivation of adults for participating in dance activities revealed that the most important reasons for adults to participate in such programs were to release everyday tension, improve physical condition, keep fit, have fun and derive pleasure from this activity and get out of home; in general, they characterized this activity as a desirable type of exercise (Papaioannidou et al., 2005; Goulimaris et al., 2008). The study by Hui et al (2009) was conducted to investigate the improvement of physical and mental well-being of elderly people in Hong Kong. The study sample comprised 111 volunteers (60-75 years old) who were divided in two groups: a) the control group, who continued their daily activities without any change and b) the dance intervention group, who attended 23 sessions of an aerobic dance program with a duration of 12 weeks. The results showed improvement of the dance group in cardiac function, stamina, general health condition and physical pain. In addition, the dance group participants claimed that the dance program helped them improve both their physical condition and psychological status as perceived by themselves. According to the study, structured dance programs are important in improving the physical and psychological health of elderly Chinese men and women (Hui et al., 2009). The study by Eyigor et al. (2009) aimed at examining the impact of a group class of traditional Turkish dances on physical fitness, balance, depression and QoL of 40 healthy women over the age of 65 . Following the implementation of the program, researchers found significant improvement in the intervention group that attended the dance program with regard to physical condition and balance, as well as to some QoL parameters such as physical functioning, general and mental health. On the contrary, the control group that did not participate in any form of physical exercise had evidence of aggravation in their general health condition with no change in any other variable (Eyigor et al., 2009). Also, Papaioannou et al. (2010) investigated the impact of the Greek traditional dance on QoL of elderly women. The results of the survey revealed that the female elderly dancing traditional dances presented a decrease in their occasional stress levels and a significant increase in their well-being. In conclusion, research has shown that engaging in Greek traditional dance leads to significant improvement of the psychological and physical well-being of elderly women and, as a consequence, to the ugrading of their QoL (Papaioannou et al., 2010).

Patients with Parkinson's disease (PD) suffer mainly from motor lesions that increase the risk of falling and lead to a lower level of QoL. Heiberger et al (2011) investigated the long-term effects of dance on QoL of PD patients and their caregivers. Following up both male and female patients throughout the intervention, the greatest improvement found was in muscle stiffness, but there was also significant improvement in hand movements, finger tapping and facial expression. The results of the questionnaires gave positive results in the dance intervention group with reference to their social life, health status, body image perception, functional mobility and daily skills. In addition, a beneficial effect was also found for career. In conclusion, dance improves QoL of both patients and their career, and therefore can lead to better therapeutic strategies as it is exciting, enjoyable and effective (Heiberger et al., 2011).

Similar findings have also derived from Schafer's Thesis (2011), who studied the effect of a creative music and dance program on elderly men and women, as well as on the nursing staff who took care of them. The results of the research showed that the implemented dance program had a positive impact on QoL of the Elderly as it offered them new experiences and knowledge, a creative means of expression, entertainment and feeling of enthusiasm. Also, the dance program had a positive effect on the culture of long-term care facilities and on the socialization between elderly residents and the nursing staff (Schafer, 2011). The study by Lykesas \& Tyrovola (2012) investigated the effect of the 
Greek traditional dance on adult women with regard to specific QoL parameters, promoting their personality features and unfolding their social behavior. The results indicated that the Greek traditional dance was an important and remarkable means of entertainment, relaxation and stress relief for the adult women. In addition, it contributed significantly to the adult women's communication, sociability, expressiveness and promotion of physical and mental health and, more generally, to the improvement of their QoL (Lykesas \& Tyrovola, 2012; Lykesas, et al., 2014; Lykesas, et al., 2017).

In her Thesis, Iordanidou (2012) examined the impact of a traditional Greek dance program on 7-year old schoolchildren with regard to psychosocial variables of emotional intelligence such as self-esteem, self-perception, emotion management, empathy and interpersonal relationships. According to the results, the students of the intervention group showed impressive improvement in their self-esteem, as well as in emotion management, empathy and interpersonal relationships (Iordanidou, 2012).

Furthermore, Sturm et al. carried out a study implementing a dance program in cancer patients under active anti-tumor treatment, with fatigue as an endpoint, to investigate the impact of dance on QoL as a holistic sports activity. The study revealed that the intervention group participating in the dance program presented significant improvement in cancer-associated fatigue, as well as in emotional and social functioning, and in physical performance (Sturm et al., 2014). The study conducted by Koch et al. (2014) upon the effectiveness of dance movement and dance in general as a therapeutic tool for the treatment of psychological problems related to health and QoL has shown that dance is effective in improving QoL and reducing clinical symptoms, such as depression and anxiety. There was also positive effect found on subjective well-being, positive mood and body image (Koch et al., 2014). The study by Bougiesi et al. (2014) investigated the effect of Greek dance on QoL in both healthy and non-healthy middle-aged and elderly people. The results showed that the Greek traditional dance improves mental health and in several cases is even more effective than other types of physical exercise. These findings link dancing to high QoL and lead to research questions about the appropriate dance style as well as the design of dance-based interventions (Bougiesi et al., 2014). In addition, Zisi et al. (2014) investigated whether elderly people participating in activities related to Greek traditional dances present different levels in their QoL than other peers who are not physically active or who do not participate systematically in other physical activities. There were 150 elderly men and women participating in the survey who were divided in three groups of 50 participants each: a) traditional dance group, b) physical exercise group, and c) sedentary life group. The results of the research demonstrated that the traditional dance group presented higher scores in all factors (Physical Functionality, Physical Role, Physical Pain, General Health Condition, Vitality, Social Functionality, Emotional Role and Mental Health) compared to the other two groups (Zisi et al., 2014). Moreover, there was a study investigating the effect of a Dance and Kinesiotherapy Program on QoL of two different groups suffering from neuro-kinetic disorders. According to the study, the intervention group with neuro-kinetic difficulties who attended the Dance and Kinesiotherapy program, improved their functional ability and general health status, reduced limitations in emotional functions and generally upgraded their QoL (Teixeira-Machado \& DeSantana, 2015). Researchers Zilidou et al. (2015) designed and implemented a Greek traditional dance program for providing physical exercise and conducted a study to investigate the effectiveness and impact of the program on the physical and mental health of the Elderly. The study sample comprised 69 healthy elderly participants (61 females/ 8 males) over the age of 60, with Mild Mental Disorder (MMD) and Mild Dementia. The results showed significant improvement in QoL variables, such as physical fitness, self-esteem and socialization, which is a protection shield for dementia and protects patients with MMD from potential depression that burdens the condition of patients with mental problems (Zilidou et al., 2015). The research group of Serrano-Guzmán et al. (2016) investigated whether a dance program improves sleep and blood pressure in a population of 67 middle-aged pre-hypertensive and hypertensive women. The dance movements were specifically designed to improve balance at different body positions by shifting the center of gravity. The main parameters measured were blood pressure, sleep quality and general QoL. According to the study results, the intervention group presented significant improvement in blood pressure, sleep quality and QoL as compared to the control group (Serrano-Guzmán et al., 2016). In her Thesis, Theocharidou (2017) looked into a combined program of Creative Dance and BrainDance that is based on Laban Movement Theory, and investigated its impact on HRQoL as perceived by male and female primary school students. The results showed that the above program enhanced their creative skills enriching them with new motor actions, upgraded their perceived HRQoL and improved almost all dimensions of the Kidscreen-52 Questionnaire (Theocharidou, 2017).

The aim of the present study was to investigate whether there are differences in HRQoL as perceived by fifth and sixth graders of two public primary schools attending two different Physical Education (PE) programs. The two schools were the Primary Schools of Sindos and Kimina, both located at rural/semi-urban areas of the Prefecture District of Thessaloniki. The Kimina School students attended a PE program focused on traditional dance, while the Sindos School students followed the PE curriculum as provided by the Ministry of Education, Research and Religious Affairs (2007). 
The question basically investigated was whether engaging in traditional dances actually helps fulfill purely educational goals and at the same time improve the students' health and well-being. This hypothesis is not unfounded since the traditional dance has been identified as a desirable type of exercise by Papaioannidou et al. (2005) and Zisi et al. (2014) in their studies investigating the main motivation for adults participating in dance activities. The same applies for adolescents as demonstrated by Doulias et al. (2005) in a similar study about adolescents' motivation for participating in dance activities. The answer to this hypothesis could come up through the investigation of health indices used for the assessment of children that are taught traditional dances. Part of this process is also the measurement of HRQoL with the use of the Kidscreen-52 questionnaire.

\section{Methodology}

\subsection{Subjects}

This study is an ex-post facto comparison of differences (Paraskevopoulos, 1993). The study sample comprised 65 fifth and sixth graders (11-12 years old) attending the primary schools of Sindos and Kimina in the Prefecture District of Thessaloniki. The students of the Sindos Primary School $(\mathrm{N}=32)$ composed the control group (group A) attending the PE "curriculum" as provided by the Ministry of Education, Research and Religious Affairs, while the students of the Kimina Primary School $(\mathrm{N}=33)$ composed the intervention group (group B) attending a program focused on "traditional dance"). The mean age of children was $11.49 \pm 0.616$ (ages ranging from 10 to 13 y.o.). The study was conducted in about the middle of the school year, that is when the children had already been taught for five (5) months two PE programs of a different content twice a week.

\subsection{Measurements}

The Kidscreen-52 questionnaire, which is widely recognized throughout Europe, was used to measure the participants' perceived HRQoL. The Kidscreen Questionnaires (The Kidscreen Group Europe 2006) are a family of self-referral tools that aim at assessing well-being and HRQoL of children and adolescents (8 to 18 years old) who are healthy or suffer from chronic diseases and have different nationality and cultural origins. The Kidscreen Questionnaires were developed over a three-year period (2001-2004) simultaneously in the 13 participating European countries (Austria, Czech Republic, France, Greece, Hungary, Ireland, Poland, Netherlands, Spain, Sweden, Switzerland, UK and Germany). This effort resulted in the creation of a valid and reliable self-referral tool that is available in three different versions for children and parents (Kidscreen-52, -27, -10) and translated in many languages. The Kidscreen-52 Questionnaire can be used in representative national and European health surveys, offering an analytical HRQoL profile that covers aspects of the children's and adolescents' physical, emotional, social and mental functionality and well-being. More specifically, the Kidscreen-52 assesses either the frequency of behavior/feelings or in some cases the intensity of attitudes (Ravens-Sieberer et al., 2005).

The Kidscreen-52 (complete version: 15-20 min to complete) covers the following ten dimensions: 1) Physical Activities \& Health (5 items), 2) Emotions (6 items), 3) General Mood (7 items), 4) Self-perception (5 items), 5) Leisure time (5 items), 6) Family \& Home life (6 items), 7) Friends (6 items), 8) School and Learning (6 items), 9) Self and others (3 items) and 10) Financial Resources (3 items). In this survey, data were collected for all the above dimensions apart from "Financial Resources" and "Family \& Home life" because these are not related to the benefits of creative dance and are therefore not relevant to this research. Moreover, due to the fact that the overall Kidscreen-52 score was not used, the removal of those two dimensions did not affect the data analysis. The Kidscreen-52 dimension scores are calculated with the use of the Rasch rating scales that were selected because they meet the model's requirements (unidimensionality of questions, homogeneity of questions and participants, sufficiency of overall score).

\subsection{Statistical Analysis}

The statistical data were analysed with the use of: a) descriptive statistical indices (Mean Values and Standard Deviations), b) t-test for independent samples and c) two way Analysis of Variance (ANOVA).

\section{Results and Discussion}

The internal consistency reliability of the scale was assessed with the use of Cronbach's Alpha coefficients. The Cronbach Alpha analysis was performed and revealed the following coefficients per dimension: "Physical Activities and Health" $\mathrm{a}=.738$, "Emotions" $\alpha=.768$, "Mood" $\alpha=.634$, "Self-perception" $\alpha=.709$, "Friends" a = .802, "SchoolLearning" $\alpha=.841$, and "Self and others" $\alpha=.467$. With reference to the dimension "Physical Activities and Health", the mean value for the control group (Sindos school students attending the program not focusing on traditional dance) was $\mathrm{M}=4.07, \mathrm{SD}=.64$, while for the intervention group (Kimina school students attending the program focusing on traditional dance) was $\mathrm{M}=4.41, \mathrm{SD}=.53$. This difference (.34) was statistically significant $(\mathrm{t}(63)=2.31, p=.024 d$ $=.58$ ). The "Physical Activities \& Health" scores of the control group, $\mathrm{D}(32)=.151, p=.061$, did not deviate significantly from normal, and neither did the scores of the intervention group $\mathrm{D}(33)=.150, p=.06$. The following 
Table 1 shows all mean values and t-test values for all dimensions of the Kidscreen-53 scale.

Table 1. Descriptive Statistical Indices and t-test values for Kidscreen-52 dimensions for the two groups

\begin{tabular}{|c|c|c|c|c|c|c|c|c|c|c|c|}
\hline \multirow{3}{*}{$\begin{array}{l}\text { Dimensions } \\
\text { Health }\end{array}$} & \multicolumn{2}{|c|}{$\begin{array}{l}\text { Program } \\
\text { without T.D. }\end{array}$} & \multirow{2}{*}{$\begin{array}{l}\text { Program } \\
\text { T.D. } \\
M\end{array}$} & \multirow{2}{*}{$\begin{array}{l}\text { with } \\
S D\end{array}$} & \multicolumn{4}{|c|}{$\mathrm{K}-\mathrm{S}$ test } & \multicolumn{3}{|c|}{ t-test \& d Cohen } \\
\hline & $M$ & $S D$ & & & $D$ & $p$ & $D$ & $\notin p$ & $t$ & $p$ & $d$ \\
\hline & 4.07 & .64 & 4.41 & .53 & .151 & .061 & .150 & .06 & 2.31 & .024 & .58 \\
\hline Emotions & 4.08 & .57 & 4.30 & .60 & .141 & .102 & .174 & .07 & 1.54 & .128 & .37 \\
\hline General Mood & 3.93 & .57 & 4.24 & .47 & .138 & .129 & .144 & .06 & 2.35 & .021 & .60 \\
\hline Self & 3.64 & .86 & 4.64 & 1.45 & 148 & .073 & .165 & .05 & 3.38 & .001 & .83 \\
\hline Leisure time & 3.72 & .75 & 3.54 & .80 & .133 & .158 & .136 & .123 & .932 & .355 & .23 \\
\hline Friends & 3.75 & .76 & 4.20 & .81 & 148 & .073 & .152 & .07 & 2.316 & .024 & .57 \\
\hline School -Learning & 3.34 & 1.00 & 4.05 & .66 & .192 & .062 & .148 & .53 & 3.370 & .001 & .84 \\
\hline Self \& Others & 4.45 & .52 & 4.77 & .50 & .173 & .092 & .180 & .052 & 2.453 & .017 & .62 \\
\hline
\end{tabular}

A first reading of findings reveals that a PE program based on traditional dance is not at all "lagging behind" a typical PE curriculum. As soon as similar results are found from other future studies investigating perceived QoL along with other factors as well, there will be sufficient evidence to support that a Physical Education program based on traditional dance may, under specific conditions and constraints, constitute a typical PE program (Papaioannidou et al., 2005; Goulimaris et al., 2008; Hui et al., 2009; Zisi et al., 2014).

Besides the general interpretation, the above Table also demonstrates that the traditional dance PE program presented higher results in perceived HRQoL in six out of the eight dimensions. More specifically, these are the dimensions of "Health", "General Mood", "Self", "Friends", "School - Learning" and "Self and Others". In particular, there was a statistically significant difference in the "Health" dimension. The impact magnitude of this difference is not great, but it should be noted that all studies in the literature review give similar findings about the Greek traditional dance in adults (Eyigor et al., 2009; Papaioannou et al., 2010; Lykesas, et al., 2010; Bougiesi et al., 2014; Theocharidou, 2017). However, special reference should be made to the difference in dimensions "Self" and "School - Learning". The differences in these domains are significant and this is probably due to the special nature of the lesson of traditional dances. More specifically, it has to do with teaching motor skills while providing a framework for correcting and improving these motor skills -a lesson that is completely different from learning other motor skills. Moreover, these specific motor skills are developed within a team spirit during the dancing process. In other words, children can self-regulate and learn the movement without being exposed as individuals. At the same time, dance provides the potential to improve motor skills because these are repeated many times during the dancing process. As a consequence, this context promotes self-esteem and learning.

In order to investigate the group and gender interaction in HRQoL, we used a two-way Analysis of Variance (ANOVA) (cf. Table 2).

Table 2. Two- way ANOVA results for the Kidscreen-52 dimensions

\begin{tabular}{llll}
\hline Dimensions & gender x group interaction & & gender \\
\hline Health & $\mathrm{F}(1,61)=3.831, \quad \mathrm{p}=.05$ & $\mathrm{~F}(1,61)=.010$, & $\mathrm{p}=.919$ \\
Emotions & $\mathrm{F}(1,61)=3.554, \quad \mathrm{p}=.064$ & $\mathrm{~F}(1,61)=.045$, & $\mathrm{p}=.832$ \\
General Mood & $\mathrm{F}(1,61)=.015, \quad \mathrm{p}=.902$ & $\mathrm{~F}(1,61)=.006$, & $\mathrm{p}=.937$ \\
Self & $\mathrm{F}(1,61)=.000, \quad \mathrm{p}=.994$ & $\mathrm{~F}(1,61)=1,391$, & $\mathrm{p}=.243$ \\
Leisure time & $\mathrm{F}(1,61)=.005, \quad \mathrm{p}=.943$ & $\mathrm{~F}(1,61)=.355$, & $\mathrm{p}=.053$ \\
Friends & $\mathrm{F}(1,61)=.241, \mathrm{p}=.626$ & $\mathrm{~F}(1,61)=.121$, & $\mathrm{p}=.729$ \\
School - Learning & $\mathrm{F}(1,61)=1.067, \mathrm{p}=.306$ & $\mathrm{~F}(1,61)=.287$, & $\mathrm{p}=.594$ \\
Self \& Others & $\mathrm{F}(1,61)=2,108, \mathrm{p}=.152$ & $\mathrm{gen}$ \\
\hline
\end{tabular}

An initial reading of the above findings reveals that there were no gender differences in any dimension, as opposed to what other studies have shown (Hui et al., 2009; Schafer, 2011; Heiberger et al., 2011; Zilidou et al. al., 2015; Theocharidou, 2017). In the traditional dance-related literature, there are gender differences found at least with regard to the participation rate in traditional dance (Graham 1995; Digelidis \& Papaioannou, 1999). As a consequence, there is need for further investigation of differences by gender.

\section{Conclusions and Suggestions}

The present study was an ex-post-facto comparison of differences in perceived HRQoL among male and female students of two public primary schools attending two different Physical Education programs: a) a typical PE program (control group) and b) a PE program focusing on Greek traditional dance (intervention team). According to the results, there were differences in perceived QoL, with the program based on Greek traditional dance presenting higher values than the PE curriculum as suggested by the Ministry of Education, Research and Religious Affairs. The implementation 
time period was limited only to five (5) months and probably a longer and more extensive program would have given different results. The proposed intervention program helped students create and discover new knowledge, significantly improve QoL variables such as physical fitness, self-esteem and socialization, enhance self-knowledge, well-being and positive mood. Finally, students of the intervention group (A) presented higher interest and more active participation in the lesson compared to the control group (B). Hence, dance, as a means of release of tension and uneasiness, as well as a pleasant physical activity at school and leisure time, contributes to the recreation and entertainment of participants offering them opportunities to express emotions and develop social relationships. Therefore, the traditional dance constitutes the most reproducible typically organized system of physical communication in the field of culture. The communicative power of dance lies in its ability to activate the whole body through the synergy of all senses, as well as to support the psychomotor, cognitive, creative and social-emotional development of students improving the relationships and communicative skills with their schoolmates (Lomax et al., 1972; Capel, 1986; Nieminem 1997; Lykesas, \& Zachopoulou, 2006; Lykesas et al., 2009; Theocharidou, 2017). However, in order to generalize such a conclusion, there is need for further research to be conducted at different schools in different grades in different regions during different time periods. It is essential to carry out further studies with the use of reliable tools for data collection and analysis, as well as mixed-type surveys. In conclusion, the Greek traditional dance provides multiple benefits to its participants, since it is not just a kind of physical activity that offers pleasure but also contributes to the improvement of Quality of Life in many domains.

\section{References}

Bougiesi, M., Gianni, A., \& Zisi, V. (2014). Quality of Life and Dance in Middle Aged and Older Adults: A Literature Review. Inquiries in Sport \& Physical Education, 12(3), 191-203.

Bullinger, M. (2003). International comparability of health interview surveys: An overview of methods and approaches. Biomedical and health research commission of the European communities, 57, 1-12.

Capel, S. (1986). Education gymnastics meeting physical education goals. Journal of Education, Recreation \& Dance, 57(2), 34-38. https://doi.org/10.1080/07303084.1986.10606041

Chen, T. H., Li, L., \& Kochen, M. M. (2005). A systematic review: How to choose appropriate health-related quality of life (HRQOL) measures in routine general practice? Journal of Zhejiang University Science B, 6(9), 936-940. https://doi.org/10.1631/jzus.2005.B0936

Chodzko-Zajko, W. J. (2005). Pychological and Sosiocultural Aspects of Physical Activity for Older Adults. In Jones C.J., Rose D.J.(Eds,), Physical Activity: Instruction of Older Adults. New ZealandQ Human Kinetics.

Cummins, R. A. (2005). Moving from the quality of life concept to a theory. Journal of Intellectual Disability Research, 49(10), 699-706. https://doi.org/10.1111/j.1365-2788.2005.00738.x

Dalkey, N. C., \& Rourke, D. L. (1973). The Delphi procedure and rating quality of life factors. In EPA, The Quality of Life Concept, $n$-209-n-221. Washington, DC: Environmental Protection Agency.

Darginidou, L., \& Goulimaris, D. (2016). Quality of Life and Traditional Dancing. Proceedings of 44th World Congress on Dance Research, July 2016, Athens, Greece.

Diggelidis, N., \& Papaioannou, A. (1999). Age-group differences in intrinsic motivation, goal orientations and perceptions of athletic competence, physical appearance and motivational climate in Greek physical education. Scandinavian Journal of Medicine and Science in Sports, 9, 375-380. https://doi.org/10.1111/j.1600-0838.1999.tb00259.x

Doulias, E., Kosmidou, E., Paulogiannis, O., \& Patsiaouras, A. (2005). Examination of Participation Motives in Folk Dance Groups. Inquiries in Sport \& Physical Education, 3(2), 107-112.

Economou, M., Kokkosis, M., Triantafillou, E., \& Christodoulou, G. (2001). Quality of life and mental health. Conceptional approaches and clinical and assessment issues. Arch.Hellen Med. 18(3), 239-253.

Eyigor, S., Karapolat, H., Durmaz, B., Ibisoglu, U., \& Cakir, S. (2009). A randomized controlled trial of Turkish folklore dance on the physical performance, balance, depression and quality of life in older women. Archives of Gerontology and Geriatrics, 48, 84-88. https://doi.org/10.1016/j.archger.2007.10.008

Goulimaris, D., Fillipou, F., Kottis, I., \& Genti, M. (The Perceived Constraints Participation Factors in Greek Traditional Dances. Science of Dance, 2, 16-30.

Graham, G. (1995). Physical education through students' eyes and in student voices, Journal of Teaching in Physical Education, 14, 364-371. https://doi.org/10.1123/jtpe.14.4.364 
Greek Ministry of Education and Religious Affairs and Pedagogical Institute. (2007) Physical Education in Elementary School and Curriculum. Administration of Physical Education, Athens: Textbook Publ..

Heiberger, L., Maurer, C., Amtage, F., Mendez-Balbuena, I., Schulte-Mönting, J., Marie-Claude Hepp-Reymond, M., \& Kristeva, R. (2011). Impact of a Weekly Dance Class on the Functional Mobility and on the Quality of life of individuals with Parkinson's disease. Front. Aging Neurosci. 3, 14. https://doi.org/10.3389/fnagi.2011.00014

Hui, E., Chui, B. T., \& Woo, J. (2009). Effects of dance on physical and psychological well-being in older persons. Archives of Gerontology and Geriatrics, 49, 45-50. https://doi.org/10.1016/j.archger.2008.08.006

Iordanidou, M. (2012). The effect of an intervention of Greek traditional dances on psychosocial variables concerning 7-year students. Master of Arts in physical activity and quality of life expressly. Department of Physical Education and Sports Sciences, Aristotle University of Thessaloniki, Greece.

Jiang, Y., \& Hessel, J. E. (2006). Associations between health-related quality of life and demographics and health risks. Results from Rhode Island's 2002 behavioral risk factor survey. Health and Quality of Life, 4, 14-24. https://doi.org/10.1186/1477-7525-4-14

Karasimopoulou, S., Deri, B., Zervoudaki, E., \& Albanidis, E. (2009). Perceptions of primary schoolchildren on dimensions of their health related quality of life. Oral Presentations of the 16th International Congress of Physical Education \& Sport. TEFAA, Komotini, 17-23.

Koch, S., Kunz, T., Lykou, S., \& Cruz, R. (2014). Effects of dance movement therapy and dance on health-related psychological outcomes: a meta-analysis. Arts in Psychotherapy, 41(1), 46-64. https://doi.org/10.1016/j.aip.2013.10.004

Lomax, A., Bartenieff, I., \& Paulay, F. (1972). Choreometrics: a method for the study of cross-cultural patterns in film. CORD Dance Research Journal, VI, 13-20.

Lykesas G., Dania, A., Koutsouba, M., \& Tyrovola, B. (2017). The Effectiveness of a Music and Movement Program for Traditional Dance Teaching on Primary School Students' Intrinsic Motivation and Self -Reported Patterns of Lesson Participation. Mediterranean Journal of Social Sciences, 8(1), 227-236.

Lykesas, G., \& Tyrovola, V. (2012). The contribution of Greek traditional dance in promoting characteristics personality and social behavior of women. Woman \& Sport, 8, 25-35.

Lykesas, G., \& Zachopoulou, E. (2006). Music and movement education as a form of motivation in teaching Greek traditional dances. Perceptual and Motor Skills, 102, 552-562. https://doi.org/10.2466/pms.102.2.552-562

Lykesas, G., Koutsouba, M., \& Tyrovola, V. (2009). Creativity as an approach and teaching method of traditional Greek dance in secondary schools. Studies in Physical Culture and Tourism, 16(2), 207-214.

Lykesas, G., Siskos, V., \& Zachariadou, Z. (2010). The Effect of a Greek Traditional Dance Teaching Programme in the Improvement of High School Students Attitude in the Classroom and their Increased Satisfaction with Physical Education Lessons. Hellenic Scientific Dance Society (EL.EP.E.X.). Electronic Journal, 5, 18-36.

Lykesas, G., Tsapakidou, A., \& Tsompanaki, E. (2014). Creative Dance as a Means of Growth and Development of Fundamental Motor Skills for Children in First Grades of Primary Schools in Greece. Asian Journal of Humanities and Social Studies, 02(01), 211-218.

Mooney, A. (2006). Quality of life: Questionnaires and questions. Journal of Health Communication, 11(3), 327-341. https://doi.org/10.1080/10810730600614094

Nieminem, P. (1997). Participation profiles and socialism into dance among non-professional dancers. Sport Education and Society, 2(2), 221-234. https://doi.org/10.1080/1357332970020206

Papaioannidou, M., Basdeki, N., \& Filippou, F. (2005). Motivations for adult participation in dance activities. Sports \& Society, 39, 69.

Papaioannou, C., Argiriadou, E., \& Mavrovouniotis, F. (2010). The effect of Greek Traditional dances on elderly women's well-being. Woman \& Sport, 7, 25-38.

Paraskevopoulos, Y. (1993). Methodology of scientific research. Editions Athena, 1, Athens.

Petraki, V., \& Koutsouba, M. (2013). Greek traditional dance as a mean of positive psychology: a review. Proceedings of the 31st World Congress on Dance Research, 1-26.

Ravens-Sieberer, U., Gosch, A., Rajmil, L., Erhart, M., Bruil, J., Duer, W., ... the European KIDSCREEN Group. (2005). KIDSCREEN-52 quality-of-life measure for children and adolescents. Expert Review of Pharmacoeconomics \& Outcomes Research, 5(3), 353-364. https://doi.org/10.1586/14737167.5.3.353 
Sandel, S., Judge, J., Landry, N., Faria, L., Ouellette, R., \& Majczak, M. (2005). Dance and movement program improves quality-of-life measures in breast cancer survivors. Cancer Nursing, 28(4), 301-309. https://doi.org/10.1097/00002820-200507000-00011

Schafer, K. K. (2011). Importance of a Creative Dance Program for the Quality of Life of Long Term Care Residents. Master of Arts in Occupational Therapy Theses. St. Catherine University, St. Paul, Minnesota, U.S.A.

Serrano-Guzmán, M., Valenza-Peña, M. C., Serrano-Guzmán, C., Aguilar-Ferrándiz, E., Valenza-Demet, G., \& Villaverde-Gutiérrez, C. (2016). Effects of a dance therapy programme on quality of life, sleep and blood pressure in middle-aged women: A randomised controlled trial. Medicina Clínica (English Edition), 147(8), 334-339. https://doi.org/10.1016/j.medcle.2016.11.016

Sturm,I., Baak, J., Storek, B., Traore, A., \& Thuss-Patience, P. (2014). Effect of dance on cancer-related fatigue and quality of life. Supportive Care in Cancer, 22(8), 2241-2249. https://doi.org/10.1007/s00520-014-2181-8

Teixeira-Machado, L., \& DeSantana, J. (2015). Dance Therapy Improves Quality of Life in Individuals with Neuromotor Disorders: Randomized Controlled Trial. International Journal of Humanities Social Sciences and Education, 2(4), 84-92.

The KIDSCREEN Group Europe (2006). The KIDSCREEN Questionnaires - Quality of life questionnaires for children and adolescents. Handbook. Lengerich: Pabst Science Publishers, http://www.kidscreen.org.

Theocharidou, O. (2017). The effectiveness of a creative dance program based on the Laban theory, relating to the quality of life perceptions associated with the health of students/three in primary education. Department of Physical Education and Sports Sciences, Aristotle University of Thessaloniki, Greece.

Tountas, Y., Tsiantis, G., Dimitrakaki, J., Petanidou, D., Tzavara, C., Diareme, St., Papadopoulou, K., \& Stoikidou, M. (2006). The kidscreen study: Assessing the quality of life related to the health of children and adolescents. In: file:///C:/Users/user/Downloads/5174675.pdf

Tyrovola, V., Koutsouba, M., \& Gratsiouni, D. (2011). Dance and physical health of older people. Rapprochement of Cultures, Proceedings of the 31st World Congress on Dance Research, 1-17.

Venetsanou, F., \& Koutsouba, M. (2015). The Study of the Dancers' Physical Fitness: Present State of Art and Future Trends. Science of Dance, 8, 1-26.

Zilidou, B., Duka, S., \& Tsolakis, M. (2009). The results of an intervention program of traditional dances, as a recreational activity, improving the quality of life of senior citizens at the Municipality of Thessaloniki. Hellenic Journal of Sport \& Recreation Management, 12(1), 13-25.

Zisi, V., Gianni, A., Bougiesi, M., Pollatou, E., \& Michalopoulou, M. (2014). Systematic Participation in Folk Dance or Physical Activity? Effects in Quality of Life in the Elderly. Inquiries in Sport \& Physical Education, 12(1), 1-8.

\section{Copyrights}

Copyright for this article is retained by the author(s), with first publication rights granted to the journal.

This is an open-access article distributed under the terms and conditions of the Creative Commons Attribution license which permits unrestricted use, distribution, and reproduction in any medium, provided the original work is properly cited. 